\title{
CyberKnife Stereotactic Radiosurgery for Cholangiocarcinoma
}

\author{
Yuko Harada $^{1}$ and Shinichiro Miyazaki ${ }^{2}$
}

\begin{abstract}
:
Cholangiocarcinoma is a devastating cancer with a poor prognosis. Patients are often diagnosed at the advanced stage, and curative surgery is impossible. Radiation therapy is used for cases of advanced cancer. A 50-year-old woman with stage IVb intrahepatic cholangiocarcinoma was diagnosed with unresectable cancer, and had chemotherapy; however, the disease progressed. CyberKnife radiosurgery was performed, which was able to control the symptoms of liver failure and prolong her life. Her symptoms, which included jaundice, ascites, and edema disappeared and her tumor markers became normal. Even though she relapsed and was not able to survive, she was able to stay home with her family until the last days of her life. CyberKnife radiosurgery was useful as a non-invasive palliative treatment and successfully alleviated the symptoms of a patient with advanced cancer.
\end{abstract}

Key words: CyberKnife, stereotactic body radiotherapy, cholangiocarcinoma

(Intern Med 57: 1411-1414, 2018)

(DOI: 10.2169/internalmedicine.9572-17)

\section{Introduction}

Cholangiocarcinoma is a rather rare cancer with a poor prognosis. Hori et al. reported that the incidence of biliary tract cancer (including cholangiocarcinoma and gallbladder cancer) in Japan was 18.4 patients per 100,000 population (1). In a retrospective study of 242 patients, the overall median survival was 15.8 months, while the median survival for stages I, II, III, and IV, was 23, 25, 14 and 4.5 months respectively (2). It is classified into 3 groups: intrahepatic, hilar, and extrahepatic. Surgical resection is the mainstay therapy; however, surgery is difficult to perform in cases of advanced-stage disease. Although chemotherapy is known to be effective, the prognosis of patients who receive chemotherapy is still poor. Radiotherapy is used to treat cases of unresectable cancer; however, conventional external radiotherapy is difficult to perform because of complications such as cholangitis, bile duct bleeding, and duodenal ulcer $(3,4)$.

The CyberKnife (Accuray Incorporated, Sunnyvale, USA) is a fully robotic radiation delivery system that can be applied as an alternative to surgery. It enables us to treat pa- tients on an outpatient basis in a much shorter period than standard radiation therapy.

We successfully treated a patient with stage IVb cholangiocarcinoma with CyberKnife stereotactic radiosurgery. The CyberKnife is a non-invasive treatment option for advanced cancer that has shown to preserve a patient's quality of life.

\section{Case Report}

A 50-year-old woman presented with jaundice and liver dysfunction. An abdominal CT scan revealed a huge tumor of $90 \mathrm{~mm}$ in diameter. Liver biopsy revealed adenocarcinoma. She was diagnosed with intrahepatic cholangiocarcinoma stage IVb (TNM stage: T4N1M1). The cancer was unresectable because it had invaded both lobes of the liver, both bile ducts and the portal vein, and multiple lymph node metastases were present around the inferior vena cava. The patient was treated with chemotherapy consisting of gemtacibine $(1,550 \mathrm{mg} /$ body $)+$ tegafur/gimeracil/oteracil $(100$ $\mathrm{mg} /$ body). The tumor mass was reduced by $15 \%$; however, it grew back after 7 months. The outcome was progressive disease (PD). She was then treated with chemotherapy con-

${ }^{1}$ Department of Internal Medicine, Shin-yurigaoka General Hospital, Japan and ${ }^{2}$ Department of Radiation Oncology, Shin-yurigaoka General Hospital, Japan

Received: May 26, 2017; Accepted: September 20, 2017; Advance Publication by J-STAGE: December 27, 2017

Correspondence to Dr. Yuko Harada, adayuko1219@yahoo.co.jp 


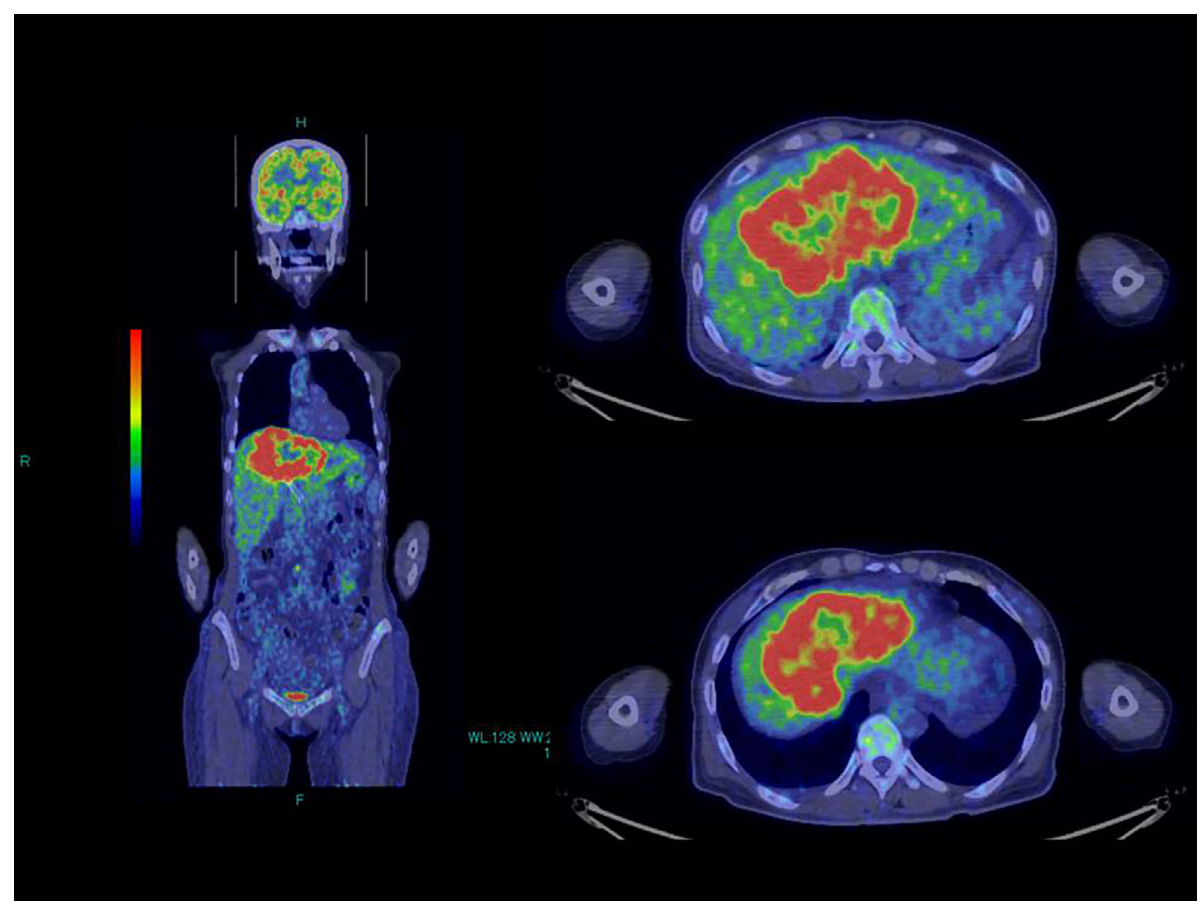

Figure 1. FDG-PET before CyberKnife radiosurgery. An enormous tumor with the uptake of FDG was observed.

sisting of gemtacibine $(550 \mathrm{mg} /$ body $)+$ cisplatin (CDDP) (38 mg/body). However she developed ascites and her jaundice and her condition worsened after 4 months. The outcome of this second treatment was PD. A stent was inserted into the bile duct. The best supportive care (BSC) was proposed; however, she wanted an alternative treatment in order to survive.

She was referred to our hospital for CyberKnife stereotactic radiosurgery. She presented with jaundice, ascites and edema in the bilateral legs. Her total bilirubin level was 5.02 (direct bilirubin, 3.48) $\mathrm{mg} / \mathrm{dL}$, despite the fact that a stent had been inserted into her bile duct 4 weeks previously. Her jaundice was likely caused by liver failure. Her carcinoembryonic antigen (CEA) and carbohydrate antigen (CA) 19-9 levels were $22.8 \mathrm{IU} / \mathrm{L}$ and $933 \mathrm{IU} / \mathrm{L}$, respectively. Abdominal CT and fluorodeoxyglucose positron emission tomography (FDG-PET) showed an enormous tumor of $107 \times 102$ $\mathrm{mm}$ in size (Fig. 1, 2). After consultations with surgeons, internists and radiotherapists, it was decided that palliative treatment was the only option. CyberKnife stereotactic radiosurgery was selected in order to provide palliative radiation therapy on an outpatient basis and to minimize the duration of treatment.

CyberKnife stereotactic radiosurgery was performed as outpatient surgery over a 10-day period. The planned tumor volume was $829.9 \mathrm{~cm}^{3}$. The prescribed dose was $40 \mathrm{~Gy}$.

One month later she visited our outpatient clinic with massive ascites and severe edema in the bilateral legs. Her CA19-9 level had decreased to 148 IU/L. She refused hospital admission and wished to stay home with her family. She was taking furosemide (40 mg/day) and spironolactone (50 $\mathrm{mg} /$ day). We increased the dose of furosemide to $60 \mathrm{mg} /$ day and sent her home according to her wishes. Two weeks later, her ascites and edema decreased, and her total bilirubin was decreased to $1.67 \mathrm{mg} / \mathrm{dL}$. We increased the prescription of furosemide to $80 \mathrm{mg} / \mathrm{day}$. Another 4 weeks later (10 weeks after CyberKnife treatment), her laboratory data normalized (total bilirubin, $1.17 \mathrm{mg} / \mathrm{dL}$; CEA, 3.8 IU/L; CA 19-9, 31 IU/L). Furthermore, she no longer had ascites or edema (Fig. 2). At four months after the treatment, FDGPET showed a significant decrease in the FDG-uptake of the tumor (Fig. 3).

She was in excellent condition for several months. Based on her condition, she decided to seek further treatment, and she started chemotherapy in another hospital (details unknown to us, but she was orally taking tegafur/gimeracil/ oteracil). She developed anemia and liver dysfunction over a 5-month period. At 10 months after CyberKnife treatment, FDG-PET showed the relapse of the original tumor and multiple lymph node metastases. She also presented with massive ascites, edema, and severe non-obstructive jaundice, and was thus admitted to our hospital, where she died of liver failure. She survived for 10 months after CyberKnife treatment.

\section{Discussion}

When the patient first visited our hospital, she had been told by her previous doctor (at another hospital) that she would survive for no longer than 3 months. The patient wanted to live as long as possible and to stay with her family in their home.

The CyberKnife is a fully robotic radiation delivery system that can be an alternative to surgery. CyberKnife radio- 


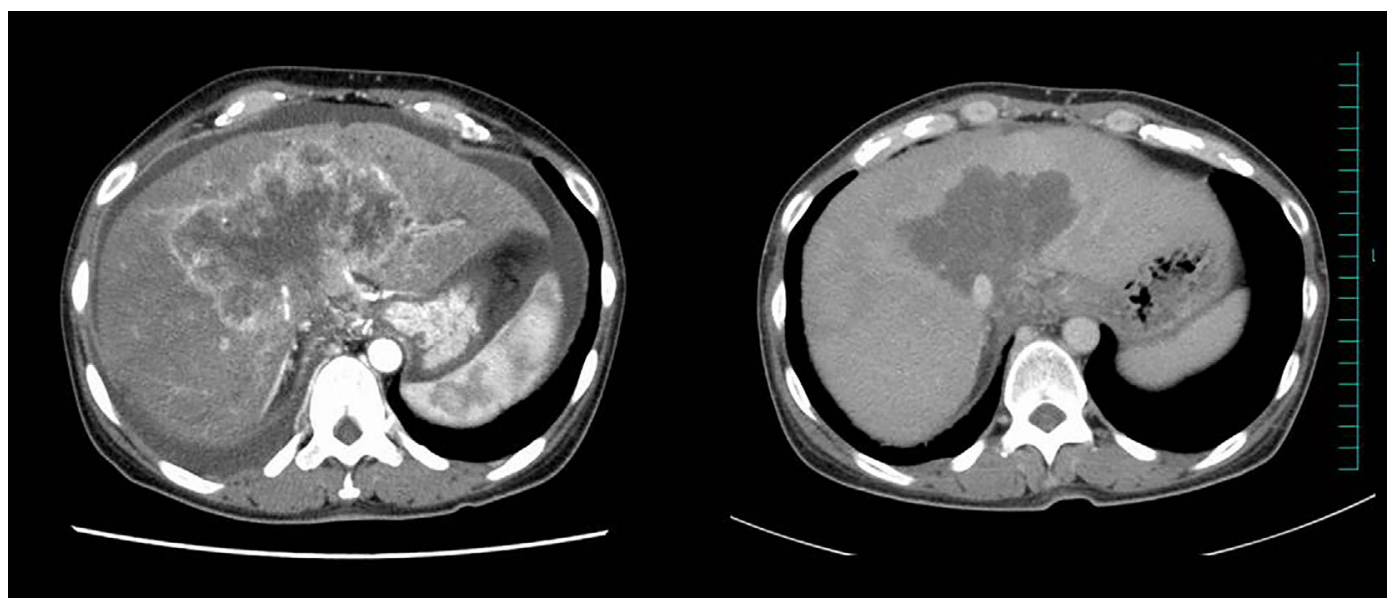

Figure 2. Abdominal CT before and after treatment. Left: Before treatment. Ascites was observed. Right: At 10 weeks after treatment, the ascites had disappeared.

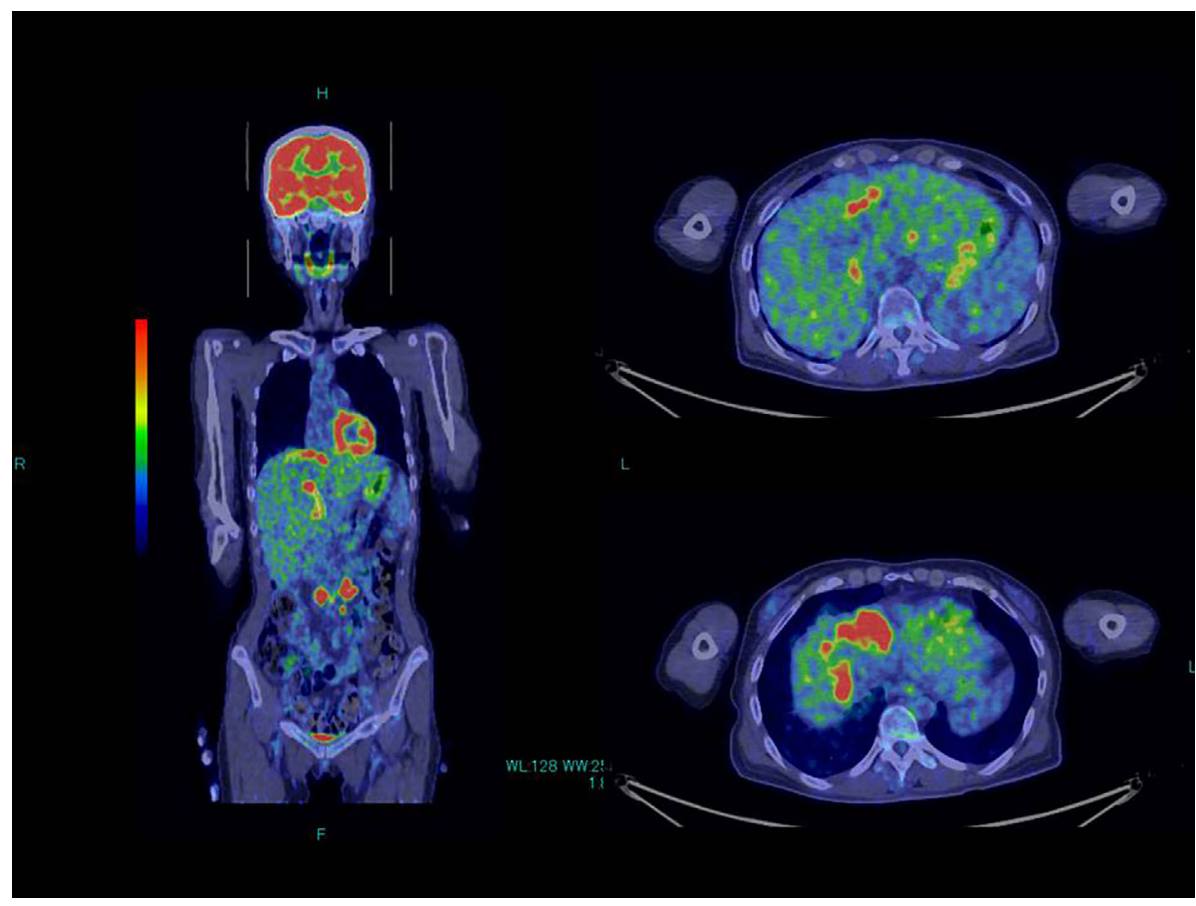

Figure 3. FDG-PET at 4 months after CyberKnife radiosurgery. The uptake of FDG was significantly decreased.

surgery is a viable option for patients with unresectable cancer. The patient still wanted to have this treatment, even though she was aware that her tumor was too large (829.9 $\mathrm{cm}^{3}$ ) for totally eradicative surgery with the CyberKnife. She underwent palliative radiotherapy with the CyberKnife, which enabled her to stay in her home for 10 months. In addition, all of her laboratory data, including her tumor marker levels normalized after the treatment, which enabled her to enjoy a normal life with her family.

The patient eventually died of liver failure, which likely occurred due to relapsed cancer or chemotherapy (tegafur/gimeracil/oteracil), because CT showed no evidence of obstruction of the bile duct, and because her laboratory data were similar to the values at the time of her first visit to our hospital. We do not believe that it was a radiation-related toxicity because it occurred 8 months after the treatment, and because we used a relatively small radiation dose for palliative treatment. To the best of our knowledge, such toxicity has not been reported as an adverse effect of CyberKnife-based treatment, and any adverse effects usually appear within several months.

In the medical literature there are only 4 previous case reports and studies of CyberKnife treatment for cholangiocarcinoma (5-8). Mahadevan et al. reported a study of 34 patients (median tumor volume: $63.8 \mathrm{~cm}^{3}$ ), in which the actuarial local control rate was $79 \%$ and the median overall survival was 17 months, with $12 \%$ of patients developing grade 3 toxicities. including duodenal ulceration, cholangitis and 
liver abscess (5). Park et al. reported a case of unresectable cholangiocarcinoma (size: $3.5 \times 4.0 \mathrm{~cm}$ ) in a patient who showed marked tumor regression at an 8-month follow-up examination (6). Dewas et al. reported the results of treatment in 6 cases of cholangiocarcinoma (median tumor volume: $32.38 \mathrm{~cm}^{3}$ ), in which the local control rate at one year was $100 \%$ (7). Jung reported on a study of 58 patients (CyberKnife treatment, $\mathrm{n}=31$; other stereotactic body radiotherapy (SBRT), $\mathrm{n}=27$; median tumor volume, $40 \mathrm{~cm}^{3}$ ), in which the local control rate at one year was $85 \%$ and the median survival was 10 months, with $5 \%$ of patients developing grade 4 complications, including cholangitis, bile duct stenosis, and gastric perforation (8). In these 4 studies, the tumor control rate was approximately $80 \%$, and the incidence of toxicities was considered minimal. However, the abovementioned median survival periods were very short and were not always prolonged by CyberKnife treatment. This is because advanced cancer with multiple metastases cannot ultimately be eradicated, even with the CyberKnife.

Conventional radiotherapy requires 4-6 weeks of daily radiation. Crane et al. revealed that tumor control improved with a higher dose (54-85 Gy) of radiation therapy, and the rates of grade $\geq 3$ toxicities were similar in all of the dose groups (14-27\%) (9). The CyberKnife is superior to conventional radiation therapy in this regard because it can deliver a high dose of radiation within a week with minimum toxicity. A large cohort study should be performed to compare conventional radiotherapy to CyberKnife radiosurgery.

In the present case report, the patient succeeded in spending her last days with her family; her life was extended and her health was far better than would have been expected if she received an alternative treatment. The CyberKnife was the only treatment option left for her, as it was a short-term treatment, and far less harmful, with measurable and favorable results. The CyberKnife has thereby again demonstrated favorable results as a successful non-invasive palliative treatment for patients with advanced cancer who seeking an al- ternative therapy that can improve their quality of life.

The authors state that they have no Conflict of Interest (COI).

\section{References}

1. Hori M, Matsuda T, Shibata A, et al. Cancer incidence and incidence rates in Japan in 2009: a study of 32 population-based cancer registries for the monitoring of cancer incidence in Japan (MCIJ) project. Jpn J Clin Oncol 45: 884-891, 2015.

2. Waseem D, Tushar P. Intrahepatic, perihilar and distal cholangiocarcinoma: management and outcomes. Ann Hepatol 16: 133-139, 2017.

3. Gonzalez Gonzalez D, Gouma DJ, Raws EA, van Gulik TM, Bosma A, Koedooder C. Role of radiotherapy, in particular intraluminal brachytherapy, in the treatment of proximal bile duct carcinoma. Ann Oncol 10: 215-220, 1999.

4. Kamada T, Saitou H, Takamura A, Nojima T, Okushiba SI. The role of radiotherapy in the management of extrahepatic bile duct cancer: an analysis of 145 consecutive patients treated with intraluminal and/or external beam radiotherapy. Int J Radiat Oncol Biol Phys 34: 767-774, 1996.

5. Mahadevan A, Dagoglu N, Mancias J, et al. Stereotactic body radiotherapy (SBRT) for intrahepatic and hilar cholangiocarcinoma. J Cancer 6: 1099-1104, 2015.

6. Park JS, Lee DH, Jeong S, et al. Concurrent chemoradiation in a patient with unresectable cholangiocarcinoma. Gut Liver 4: 103105, 2010.

7. Dewas S, Bibault JE, Mirabel X, et al. Prognostic factors affecting local control of hepatic tumors treated by stereotactic body radiation therapy. Radiat Oncol 7: 166, 2012.

8. Jung DH, Kim MS, Cho CK, et al. Outcomes of stereotactic body radiotherapy for unresectable primary or recurrent cholangiocarcinoma. Radiat Oncol J 32: 163-169, 2014.

9. Crane $\mathrm{CH}$, Macdonald $\mathrm{KO}$, Vauthey JN, et al. Limitations of conventional doses of chemoradiation for unresectable biliary cancer. Int J Radiat Oncol Biol Phys 53: 969-974, 2002.

The Internal Medicine is an Open Access article distributed under the Creative Commons Attribution-NonCommercial-NoDerivatives 4.0 International License. To view the details of this license, please visit (https://creativecommons.org/licenses/ by-nc-nd/4.0/).

(C) 2018 The Japanese Society of Internal Medicine Intern Med 57: 1411-1414, 2018 